\title{
ESPAÑA 2000, ¿LA EMERGENCIA DE UNA NUEVA DERECHA RADICAL POPULISTA
}

\author{
Anna I. López Ortega ${ }^{1}$ \\ Universitat de València
}

http://dx.doi.org/10.5209/NOMA.55258

\begin{abstract}
Resumen: ¿Es España 2000 un partido político de extrema derecha? La formación valenciana se crea en 2003 y se autodefine como "social patriota" y anti inmigración. En el 2007 obtuvo más de 12.000 votos y 5 concejales y desde 2016 se ha unido en coalición con el partido Plataforma per Catalunya. Los medios de comunicación la han definido como "neonazi" y "fascista", sin embargo no existen estudios formales sobre la ideología del partido.

Por ello, a partir del modelo de Derecha Radical Populista desarrollado por Cas Mudde se realiza un trabajo de análisis cualitativo y cuantitativo del programa electoral de E2000 de las elecciones autonómicas en la Comunidad Valenciana en 2015 y se plantea su posible catalogación dentro de una de las dos corrientes: extrema derecha o derecha radical populista. A continuación, se mide el grado de similitud con los partidos neopopulistas europeos a través de un análisis cuantitativo de los términos asociados a estas formaciones y elaborado por Antón Mellón. El resultado determinará si las posibles semejanzas ideológicas con la derecha radical populista posibilitarían el éxito del partido como ha sucedido con otras fuerzas europeas.
\end{abstract}

Palabras clave: nueva extrema derecha, ideología, partidos políticos, derecha radical populista, anti inmigración

Abstract: Is España 2000 an extreme right-wing political party? The valencian formation was created in 2003 and defines itself as "social patriot" and anti-immigration. In 2007 it obtained more than 12,000 votes and 5 councilors and since 2016 has united in coalition with the party Plataforma per Catalunya. The media have defined it as "neoNazi" and "fascist," however there are no formal studies on party ideology. For this reason, from the model of Radical Populist Right developed by Cas Mudde is carried out a qualitative and quantitative analysis of the electoral program of E2000 of the autonomic elections in the Comunidad Valenciana in 2015 and it is proposed its possible cataloging within one of the Two currents: extreme right or populist radical right. Next, the degree of similarity with the European neopopulist parties is measured through a quantitative analysis of the terms associated with these formations and elaborated by Antón Mellón. The result will determine if possible ideological similarities with the Radical populist right would make possible the success of the party as it has happened with other European forces.

Keywords: New extreme right, ideology, political parties, populist radical right, anti immigration

I Doctoranda en Estudios Jurídicos, Ciencia Política y Criminología de la Universitat de València. Investigadora en el Instituto de Ciencias Sociales y de la Cultura de la Diputació de Valencia 


\section{Introducción}

El partido político España 2000 (E2000) autodefinido como "social patriota" y "anti inmigración" nace en 2003 en Valencia. La formación ha sido objeto de estudio desde los medios de comunicación por la figura de su presidente, José Luis Roberto, vinculada a empresas de prostitución, seguridad y gimnasios. Esta imagen quizás haya disuadido a la comunidad académica de estudiar los resultados electorales del partido, su discurso e incluso la propia consideración de E2000 como partido político. De hecho, se ha llegado a calificar a E2000 como movimiento, organización o banda de ultras y neonazis. No obstante, es innegable cierto avance electoral del partido en la Comunidad Valenciana y su implantación municipal, sobre todo a partir de las elecciones autonómicas de 2007 (12.000 votos y 28 sedes locales) momento en el que el partido asume un discurso anti inmigración, islamófobo y ultranacionalista. Igualmente el partido ha renunciado a una posible identificación con el régimen fascista y ha eliminado los símbolos franquistas en sus manifestaciones. Asimismo ha establecido contactos internacionales a través de reuniones y encuentros puntuales con otras formaciones europeas de extrema derecha (Frente Nacional Francés, Vlaams Belang, Fourza Nuova). Además, su reciente alianza con el partido Plataforma per Catalunya en abril de 2016 en la coalición Respeto parece indicar que este partido político podría formar parte de familia la derecha radical populista europea, o al menos, que comparte elementos discursivos con esta corriente ideológica cuyo ascenso electoral en muchos parlamentos europeos es innegable desde 20142.

Teniendo en cuenta esta perspectiva, el artículo parte de la hipótesis de que España 2000 forma parte de la familia de la extrema derecha y probablemente el análisis de sus principios ideológicos permitirá definirla en la corriente de la derecha radical populista formulada por Cas Mudde. Sin embargo, este primer enfoque no es suficiente para corroborar la hipótesis porque aunque la clasificación de partidos de Cas Mudde es dicotómica (extrema derecha o derecha radical populista), el discurso de la formación presenta matices y por ello, se propone evaluar en qué medida el partido presenta un programa político similar al de la Derecha Radica Populista y tos principales conceptos asociados a ésta.

\footnotetext{
2 En mayo de 2014 en Parlamento Europeo, el Frente Nacional en Francia logró un $25,40 \%$ y 23 escaños, Cinco años antes había logrado un 6,3\% 3 escaños. En el Reino Unido, el UKIP (Partido por la Independencia del Reino Unido) logró, frente al 16,09 \% de 2009, un $29 \%$ y 24 escaños en esta elecciones europeas. En Austria el FPO pasó del $12,71 \%$ al 20\%. En Dinamarca, el Partido Popular (DF) duplicó apoyo electoral (del 14 al $27 \%$ ), en Letonia el partido Todo para Letonia (VL) en coalición, consiguió el $14 \%$. Otras formaciones europeas radicales lograron una mejora de sus resultados electorales en sus respectivos Parlamentos: Alemania (NPD y AfD), Chipre (Frente Nacional Popular), Croacia (HSP en coalición), Finlandia (Verdaderos Finlandeses, VS), Hungría (JOBBIK), Grecia (Amanecer Dorado).
} 
Partiendo de estas premisa se tratará de responder a la cuestión, ¿puede ser homologable su discurso de E2000 al de otras formaciones neo populistas europeas? Si es así, el resultado de la investigación permitirá reforzar la propuesta inicial de considerar a E2000 como un partido político de la familia de la extrema derecha.

El estudio se estructura en dos apartados. En el primero se desarrolla el marco teórico de la investigación a través de una breve revisión de los principales teóricos (Piero Ignazi, Hans-Georg Betz, Cas Mudde) que han abordado las características y diferencias de los partidos de extrema derecha. No se trata de profundizar en la discusión sobre las corrientes de pensamiento definitorias de este tipo de formaciones, pero sí establecer un marco ideológico mínimo para identificar a E2000, por primera vez, dentro de la familia de la extrema derecha y en concreto, la tipología concreta de extrema derecha del partido según la ideología de su último programa electoral (2015).

Para analizar los principales elementos discursivos-ideológicos del programa del partido valenciano, se ha utilizado un análisis de contenido clásico a partir del modelo de extrema derecha contemporánea de Mudde. El autor alemán propone una serie de parámetros que identificarían a una formación política como de derecha radical, estos se refieren tanto a su ideología como a sus políticas: nacionalismo exclusionista, racismo cultural, xenofobia, aversión a los procesos democráticos y la concepción de un Estado fuerte. Estos elementos permiten la adscripción a la ideología básica y a una familia unificada de partidos de derecha radical, a pesar de la heterogeneidad de los partidos.

En segundo lugar, se pretende evaluar y comparar el programa político de E2000 con el de los partidos neo populistas europeos según la propuesta de Antón Mellón desarrollada en la investigación "Inmigración y discurso electoral en las elecciones municipales de 2007 en Cataluña. Análisis de la similitud y divergencia en relación al discurso xenófobo de la Derecha Radical Europea Neopopulista". Este trabajo elabora un árbol conceptual de los discursos xenofóbicos de los partidos neopopulistas que permite analizar el grado de similitud del partido valenciano con el de los europeos. Partiendo de los conceptos formulados en su trabajo, se aplica la metodología de análisis de contenido, pero en este caso, de tipo cuantitativo ya que el programa político se ha analizado a través de una serie de codificadores contenidos en un libro de variables excel. Estas variables se han establecido de acuerdo a los conceptos atribuidos por Antón Mellón a la derecha radical. En este libro de códigos, se han cuantificado los conceptos coincidentes con la derecha radical populista y el grado de proporcionalidad. 
A partir de este estudio, se plantea una conclusión final sobre las características ideológicas del partido político E2000 y su adscripción a la familia de la extrema derecha europea y a los partidos neo populistas europeos. Asimismo, se reflexiona sobre el futuro de la formación en el contexto político y social de una Unión Europea donde la derecha radical populista ha alcanzado sus mejores cuotas electorales desde la II Guerra Mundial. La importancia del análisis de esta tendencia electoral en la Comunidad Valenciana — -si se confirma la hipótesis- reside en sus implicaciones ideológicas, culturales, políticas y sociológicas para la sociedad valenciana. Igualmente se intentará responder a la cuestión ¿este discurso de la formación valenciana facilitaría su crecimiento electoral?

Las fuentes que se utilizan en la primera parte proceden de la literatura sobre partidos políticos de extrema derecha europeos (Mudde, Ignazi, Betz) y españoles (Casals, Antón Mellón, Hernández Carr, Rodríguez). La unidad análisis de todo el artículo es la misma: el programa electoral del partido para las elecciones autonómicas en la Comunidad Valenciana del 25 de mayo de 2015, pero la unidades de medida son diferentes (párrafos y palabras respectivamente). La elección de los programas electorales como unidad de análisis de contenido en los apartados se justifica por dos motivos: el programa electoral se considera el documento que proporciona la visión general de lo que plantea el partido político en un momento determinado en el tiempo. En la mayoría de los casos, los políticos se sienten obligados a cumplir las promesas políticas establecidas en una elección. Además como un documento oficial, será difícil para los miembros del partido retractarse de las políticas contenidas en él. La segunda, más práctica, es que programas electorales son los documentos mayoritariamente aplicados para un estudio clásico en el ámbito de la Ciencia Política

\section{Una aproximación teórica a los partidos políticos de extrema derecha}

Existe gran literatura académica acerca del concepto de partido político (Weber, 1999; Sartori, 1980) y su estructura (Duverger, 1989), pero para poder identificar el objeto de estudio, E2000, y corroborar la hipótesis partimos de dos consideraciones necesarias para poder comparar al partido valenciano con otras formaciones neopopulistas: es un partido político y es de extrema derecha.

Sobre la primera cuestión, es innegable que España 2000 es un partido político, ya que es una formación política registrada en mayo de 2003 de acuerdo a la legislación española vigente de partidos políticos y contenida en el ordenamiento jurídico español. En ese sentido, el artículo 6 de la Constitución española señala que:

Los partidos políticos expresan el pluralismo político, concurren a la formación y manifestación de la voluntad popular y son instrumento 
fundamental para la participación política. Su creación y el ejercicio de su actividad son libres dentro del respeto a la Constitución y a la ley. Su estructura interna y funcionamiento deberán ser democráticos.

Destaca la importancia y trascendencia de su estudio como partido político, en el sentido de que estos son considerados la columna vertebral de cualquier democracia y constituyen opciones en las que la población basa sus preferencias para ser gobernados. Su objetivo es influenciar en la toma de decisiones ganando cargos de elección y participar activamente o no en los procesos electorales.

En segundo lugar, ¿̇es E2000 un partido de extrema derecha? La última década del siglo XX de la Europa occidental se ha caracterizado por el surgimiento de nuevas formaciones denominadas "derecha radical", "extrema derecha", "derecha populista", la "derecha autoritaria" o "nueva derecha radical". Los temas que estos partidos han utilizado para definirse incluyen la crítica de la inmigración del Tercer Mundo, las ideas de multiculturalismo, la corrupción entre las élites políticas tradicionales y su incapacidad para resolver los problemas de los ciudadanos, la carga fiscal excesiva, el Estado y la profundización de la integración europea. Estos temas se han acompañado de la explotación del nacionalismo y el tradicionalismo moral.

La aparición de estos partidos condujo a un creciente interés entre los científicos políticos. La investigación, sin embargo, estuvo marcada por ideas muy diferentes sobre estos partidos según los especialistas, el país y partido analizado. El principal problema estaba relacionado con los diferentes enfoques conceptuales que se han desarrollado en esta área. Precisamente es el tema de la conceptualización de la extrema derecha el que abordamos a continuación. Para ello, se resumen los enfoques básicos para el estudio de este fenómeno político. Dado que el objeto de estudio no es el debate de las diversas propuestas, este apartado no será exhaustivo, sino más bien una introducción selectiva de algunos de los conceptos más conocidos para aplicarlos a la ideología de E2000.

Las cuestiones a resolver son dos: zes posible considerar a E2000 dentro de la familia de la extrema derecha? Y si es así, ¿̇es posible homologar su discurso al de los partidos neopopulistas europeos?

\section{La influencia del fascismo en la extrema derecha}

En la primera mitad del siglo XX, los partidos políticos de extrema derecha han estado ligados al fascismo. Sus fundamentos ideológicos, sin embargo, eran bastante imprecisos, a menudo eclécticamente combinados con las ideas de otras ideologías. Sus principales defensores eran muy conscientes de ello. Como Mussolini señaló, "[...] el fascismo 
difiere de otros programas en su espíritu [...], que se basa en la guerra y la victoria" (O'Sullivan, 1995: 195).

Sin embargo, los autores que analizan el fascismo coinciden en varias características generales que son indicativas de ambas ramas del fascismo, el italiano y el alemán (Fritzsche, 1977: 470; O'Sullivan, 1995; 137; Heywood, 2004: 209; Feldman, 2006: 455; Griffin, 2006: 446). El fascismo se caracterizó por la negación del racionalismo, el progreso, la libertad y la igualdad, y generalmente todo lo relacionado con "el año 1789", a excepción del activismo político, que se hizo importante para el fascismo. El fascismo también se caracterizó por el rechazo del capitalismo, el tiberalismo, el comunismo, la democracia y el sistema parlamentario; defendió la idealización de la nación y la lucha como impulso fundamental de la historia. También fueron cruciales la aplicación del principio de liderazgo y el culto al líder, el heroísmo y el corporativismo que funcionaban dentro de la sociedad orgánica.

Esto está relacionado con las ideas sobre la creación de un "hombre nuevo", inmerso en una revolución permanente como fundamento de la dinámica social, adoptando una visión mesiánica del mundo. El Estado es una proyección de la unidad clave que reemplaza a los intereses del individuo y una expresión de unidad nacional y autarquía nacional. También es imprescindible el papel del mito (diversamente comprendido) que aparece, por ejemplo, en la idea italiana de completar el resurgimiento y en el concepto alemán de la creencia en la necesidad de la lucha por la regeneración de la Volksgemeinschaft. La versión alemana (nacionalsocialista) del fascismo difería del italiano por su énfasis en la raza y el principio racial (división en razas "superiores" e "inferiores") y su antisemitismo.

En la primera mitad de la década de 1980, el politólogo Klaus von Beyme en su obra Parteien in westlichen Demokratien fue uno de los primeros autores en describir el concepto de familias en el espectro político de la extrema derecha. Este autor determina que los partidos circunscritos en la extrema derecha se refieren al fascismo, tanto en sus formas italianas y alemanas entre guerras, como en sus diversas mutaciones $y$ restos influenciados por las condiciones nacionales y específicas del período. Estas mutaciones y remanentes estaban a menudo, por supuesto, como decía von Beyme, bastante alejados de los dos modelos principales $y$, fundamentalmente en algunos aspectos, divergentes. Por ejemplo, sobre el contexto español, Von Beyme escribió:

El movimiento español fue siempre una mezcla de elementos fascistas (Falange) y tradicionalistas con astillas de derecha sindicalistas. El partido era sólo una percha del movimiento militar bajo Franco. Después del éxito del golpe militar, los fascistas obtuvieron más apoyo como organización veterana (...), pero para el dolor de los viejos falangistas se controló su ardor fascista .(Von Beyme, 1985: 125). 
Durante el transcurso de las siguientes décadas después de 1945, varios grupos neofascistas estaban presentes en la periferia política y social en los regímenes occidentales democráticos. Era legales pero su importancia electoral era insignificante. La única excepción aislada fue el Movimiento Socialista Italiano post-Mussolini. Von Beyme también trató de manera limitada con el surgimiento de nuevos partidos de movimiento de protesta cuya relación con la tradición fascista ampliamente entendida era insignificante y difícilmente podrían considerarse tendencias totalitarias 0 ser tomadas como manifestaciones del neofascismo. Se trata, por ejemplo, del Poujadismo en Francia en los años cincuenta, basado especialmente en la defensa de los "autónomos" ordinarios, los propietarios de pequeñas empresas y los agricultores contra las grandes empresas y la burocracia estatal, o ambos Dinamarca y Norvega (los Fremskridtsparti y los Fremskrittsparti) desde los años setenta, cuando protestaron principalmente contra el exceso de impuestos. Von Beyme reconoce la necesidad de un concepto más amplio del extremismo derechista. Sin embargo, en su obra no hubo un nuevo reexamen importante de la familia de partidos de extrema derecha, probablemente por el escaso y volátil apoyo electoral de estas formaciones durante esa época.

Desde la década de 1960 muchos autores han señalado el impacto de los cambios sociales y económicos dinámicos sobre los valores y la orientación política de los votantes. Especialmente conocida fue la idea de Ronald Inglehart (1971 y 1990) de que una "revolución silenciosa" introdujo un gradual cambio de valor post-materialista a una generación joven, educada y materialmente segura, y contribuyó al surgimiento de los partidos ecológicos y de otros partidos libertarios de izquierda a finales del siglo XX.

Piero Ignazi contradice a Inglehart $y$ determina que existe una "contrarrevolución silenciosa". En su opinión, la expansión actual de la extrema derecha es un cierto tipo de reacción social en las sociedades postindustriales. La economía mundial exige una mano de obra mucho más móvil y flexible. Sin embargo, una gran parte de la sociedad ha tenido dificultades para adaptarse a los cambios. Se encuentran a menudo en una posición marginalizada caracterizada por el desempleo a largo plazo, la creciente frustración y privación, y un anhelo de renovación del statu quo anterior con sus relaciones, vínculos, orden y armonía tradicionales. Además, la afluencia de solicitantes de asilo e inmigrantes del Tercer Mundo en Europa Occidental en las últimas décadas han despertado actitudes de xenofobia y racismo. Este contexto crea un ambiente favorable para fuerzas políticas radicales y líderes carismáticos que promete superar la creciente atomización de la sociedad, rechazan el post-materialismo y aseguran resolver rápidamente los problemas del desempleo, la inmigración, la 
criminalidad y otras demandas de los votantes que se sienten amenazados.

De ahí que de los partidos tradicionales más afectados por el ascenso de la extrema derecha fueran en una primera etapa los partidos socialdemócratas. Sus votantes de la clase obrera están entre aquellos para quienes la adaptación a las circunstancias cambiantes ha sido más difícil (Kitschelt, 1994).

\section{El debate nominativo}

En este contexto diversos autores estudian a los partidos de extrema derecha y sus diferentes análisis de enfoques conceptuales ha provocado la aparición de un debate terminológico. Sin embargo, existe una mayor unanimidad entre los autores (Ignazi, 1992: Mudde, 2000: Holsteyn, 2001) en diferenciar dos corrientes: la "extrema derecha" o "vieja extrema derecha" de la "nueva extrema derecha" o "derecha radical populista".

Los especialistas alemanes Backes y Jesse diferencian entre "extremismo" y "radicalismo". Mientras que el término "extremismo" se asocia con el rechazo total de la democracia liberal, y los objetivos antiparlamentarios y anti-constitucionales, -un término con connotaciones negativas-, los términos "radicalismo" y "radical" reflejan que son más un camino de acción que puede o no ser antidemocrático. No obstante, para la escuela de pensamiento alemana, el concepto "derecha radical" se percibe como una categoría transitoria entre la "extrema derecha" y el "moderado conservador, democrático cristiano" (Mares, 2003: 21).

La escuela anglosajona utiliza el concepto de "derecha radical" (Ramet, 1999; Griffin, 2000; Norris, 2005) pero como un significado más complejo y diferente respecto al de los expertos alemanes. Esta escuela está influenciada por la antigua tradición del nativismo americano (sentimiento anti-inmigración), el populismo y la hostilidad al gobierno central, combinados con ultra-nacionalismo, anticomunismo, fundamentalismo cristiano y orientación militarista (Mudde, 2000: 12).

Piero Ignazi plantea tres criterios para clasificar a los partidos de extrema derecha. En primer lugar, para ser considerado extrema derecha, los partidos deben situarse en el extremo derecho del continuum izquierdaderecha, sin ninguna posibilidad de ubicación más a la derecha. Segundo, debe tener un vínculo ideológico con la mitología y los principios fascistas (ultranacionalismo, el antipluralismo, una concepción autoritaria del orden social). En tercer lugar, debe expresar valores, cuestiones y políticas que rechacen y deslegitimen el sistema democrático. 
El primer criterio es generalmente reconocible, aunque se podría plantear la cuestión de cómo clasificar dos o más partidos en el mismo país que se encuentran a la derecha de la derecha moderada. Esto podría resolverse incluyéndolos en la familia de los partidos de extrema derecha, pero señalando las diferencias entre ellos. Por lo tanto, un partido es considerado extrema derecha según Ignazi si cumple al menos el primer y el tercer criterio, es decir, debe ser una formación anti sistema. En este contexto se refiere al concepto tradicional de Giovanni Sartori (1976) de un partido anti-sistema, como oposición de principio al sistema político como tal.

Sobre esta base Ignazi divide a la extrema derecha en dos grupos. El primer grupo se compone de los antiguos partidos tradicionales, de "vieja extrema derecha" que mantienen vínculos fundamentalmente ideológicos con los movimientos fascistas de entreguerras, con propuestas radicales y objetivos definidamente antidemocráticos. La vieja extrema derecha preconiza la instauración de un orden corporativo y recurre a mecanismos de representación no individuales que tienen por lo menos vínculos mínimos con el fascismo. Se trata de formaciones que han actuado en el transcurso de las tres últimas décadas pero con la derrota de los fascismos en 1945 o la nostalgia de las dictaduras anticomunistas en la Guerra Fría (el franquismo, el salazarismo o el régimen de los corones griegos) han desembocado en la marginalidad $y$ en el declive generalmente, excepto aquellos partidos que se han renovado como es el caso del Movimiento Social Italiano y su reconversión en 1995 en Alianza Nacional. No obstante, podemos englobar como partidos de esta tradicional extrema derecha al Partido Nacional Demócrata Alemán, British National Party, National Front inglés, Unidad Nacional de Grecia, Falange Española Auténtica de las JONS y Círculos Doctrinales de José Antonio.

Estos movimientos tienden a la marginalidad en los países europeos más desarrollados $y$ en muchos casos se encuentran fuertemente atomizados. En cambio, Ignazi utiliza el término "nueva extrema derecha" para referirse a los partidos de reciente creación, ubicados dentro de la corriente de la "derecha postindustrial" (Ignazi, 1992: 372).

De esta manera, estos partidos se caracterizarían por intentar responder a los problemas de la sociedad actual sin identificarse claramente con el fascismo histórico (ultranacionalismo, anti pluralismo, concepción autoritaria del orden social). Estas formaciones han cosechado un espectacular éxito desde mediados de los noventa hasta nuestros días gracias a un discurso que se aleja del fascismo histórico y que pretende responder a los retos de la sociedad actual sin identificarse claramente con el pasado. Ignazi data su nacimiento en 1992 debido al proceso de "contrarrevolución silenciosa" en la que convergen el impacto del neoconservadurismo, la tendencia de la polarización política y la pérdida de legitimidad de los sistemas democráticos como 
consecuencia del discurso crítico efectuado por los partidos conservadores.

Se trataría de partidos como el Frente Nacional en Francia, el Fremskrittsparti (Partido del Progreso) en Norvega, o el Dansk Folkeparti (DF, Partido del Pueblo Danés).Sin embargo, surge una duda acerca de algunas de las partes que Ignazi califica como anti-sistema, su tercer criterio para pertenecer a la familia del partido. Tomemos los ejemplos de la Alleanza Nazionale y del Freiheitliche Partei Österreichs de Austria (FPÖ, Partido de la Libertad). Ambos participaron en el gobierno durante períodos significativos a comienzos del siglo XXI sin tratar de destruir el régimen democrático. A pesar de sus opiniones radicales, se puede dudar de que cualquiera de estos partidos tenga un objetivo antidemocrático y se esfuerce por deslegitimar el Régimen en el sentido de la oposición de principio de Sartori. Ignazi sostiene que los partidos de extrema derecha a menudo "muestran incompatibilidad de objetivos y aceptabilidad de comportamiento"(2003: 32).

No obstante, es necesario tener en consideración el actual contexto político, que es diferente del que prevalecía durante la Guerra Fría cuando Sartori acuñó el término partido anti-sistema. Durante la era posterior al final de la Guerra Fría se produjo una transformación en muchos de los antiguos partidos anti-sistema. Este fue un proceso experimentado no sólo por el MSI en Italia, sino por un número de ex partidos comunistas. En general, el resultado ha sido que al menos algunos de los partidos de extrema derecha sólo pueden ser considerados como partidos de protesta o anti-establishment (Schedler, 1996; Kubát, 2007). En consecuencia, la actitud de los partidos de extrema derecha hacia el régimen democrático liberal puede abarcar desde el estricto rechazo hasta la postura meramente crítica de ciertos fenómenos negativos que son el funcionamiento actual de varios regímenes democráticos contemporáneos (corrupción, excesiva mezcla de las esferas política y económica).

Una definición menos rígida y mucho más amplia de la extrema derecha que la de Ignazi es ofrecida por Hans-Georg Betz (1993), que utiliza el término de "partidos populistas de extrema derecha". Según Betz, estos partidos se oponen radicalmente al sistema cultural y sociopolítico actual en las democracias occidentales, pero sin atacar directamente sus fundamentos. Rechazan la igualdad individual y social y enfatizan la homogeneidad cultural o étnica en la sociedad, con una preferencia por "nuestro propio pueblo" respecto a los "extranjeros". Es decir, en su mayor parte, inmigrantes. A menudo hacen hincapié en la economía neoliberal, pretenden defender a la "persona común" contra el establishment corrupto y los órganos del Estado. Destacan el "sentido común" y la ley y el orden, son antifeministas y se consideran defensores de los valores tradicionales. Organizacionalmente, a menudo son dirigidos por una figura popular o incluso carismática (Jean-Marie Le Pen 
y el Frente Nacional, Pia Kjaersgaard y el Dansk Folkeparti, etc.). Están altamente centralizados y jerárquicamente estructurados, usan el populismo para obtener votos y son capaces de hacer uso efectivo del marketing político. Sin embargo, el enfoque de Betz no es tanto una definición ideológica como una descripción de su estilo político.

En la práctica esto supone un problema para elaborar una familia de partidos de extrema derecha, aunque debe considerarse una contribución a su caracterización general. Meindert Fennema contempla la extrema derecha, o una gran parte de ella, desde la perspectiva de la oposición a la inmigración. Esto se refleja en su uso del término de trabajo de partidos anti-inmigrantes. Sin embargo, como se demostró anteriormente, la inmigración representa un tema muy común en la extrema derecha, pero para la mayoría de estos partidos, éste no es el gran problema. Los autores anteriores trataron de resolver la cuestión al referirse a la inmigración como una "super-cuestión". Sin embargo, su definición de los partidos anti-inmigrantes es demasiado vaga para encajar dentro de su definición básica de la familia del partido de extrema derecha. Estos autores los definen como partidos que han intentado movilizar votos sobre la base de sentimientos antiinmigrantes, y son estigmatizados por los partidos políticos principales (Brug et al 2000: 83). Uno de los aspectos dudosos de la primera condición es que la estrategia de movilizar a los votantes al exigir una regulación estricta de la inmigración también ha comenzado a ser utilizada por algunos partidos de derecha moderados.

Como otro enfoque posible para estudiar a la extrema derecha europea, analizamos la propuesta del politólogo holandés Cas Mudde ya que probablemente se haya convertido en el modelo más aceptado en la literatura especializada. Su planteamiento permite extraer una serie de características que identificarían a la "derecha radical populista" y, así, poder aplicarla a un determinado partido político.

En primer lugar, dentro de lo que este autor considera "familia de partidos", distingue entre la "derecha radical populista" y "extrema derecha". Su principal diferencia, reside en que la primera "puede considerarse democrática aunque se oponga a algunos valores fundamentales de las democracias liberales, mientras que la extrema derecha es en esencia anti-democrática, al oponerse al principio fundamental de la soberanía del pueblo" (Mudde, 2007: 34).

Para Mudde los partidos de "Derecha Radical Populista" comparten un núcleo ideológico común que incluye la combinación de las siguientes características: nacionalismo exclusionista, racismo cultural, xenofobia, aversión a los procesos democráticos y la concepción de un Estado fuerte. Estas conclusiones son producto del trabajo que desarrolla Mudde tras analizar las teorías de Daniel Bell sobre la derecha radical en Estados Unidos en los años cincuenta con el objetivo de recordar 
cómo esta corriente se ha considerado en los estudios sociológicos como una patología producida por las crisis:

Casi todas las grandes teorías sobre el apoyo a la derecha radical populista, en el esquema de la tesis de patología normal, se refieren a alguna forma de crisis ligada a la modernización y a sus consecuencias: globalización, economía postfordista, sociedad postindustrial. La idea es siempre la misma, la sociedad se transforma rápida y fundamentalmente dando lugar a una división (auto percibida) entre 'vencedores' y 'perdedores', los últimos votan por la derecha radical populista como protesta (enojo y frustración) o como apoyo (rigidez intelectual). Bajo condiciones de cambio social masivo, los perdedores de la modernización van a votar por partidos de la derecha radical populista (Mudde 2010: 2).

Sin embargo, este autor propone otro paradigma interpretativo al que denomina "normalidad patológica". Plantea que la extrema derecha populista europea no es la antítesis de la democracia liberal sino una radicalización de los valores dominantes en el seno de las masas. Sus tres rasgos fundamentales serían el nativismo, populismo y autoritarismo. En el caso del "nativismo", estamos ante una ideología que sostiene que la nación debe ser habitada exclusivamente por miembros del propio grupo. En cuanto al "autoritarismo" y el "populismo", defiende la pureza del pueblo frente a las élites corruptas. Así, bajo el discurso de los derechos, destaca el derecho a la diferencia y a la propia cultura, y proponen un sistema de exclusión que pretenden validar democráticamente, de modo que el sistema acepte y adopte sus posturas discriminatorias y excluyentes. Los sentimientos anti establishment del populismo están estrechamente conectados con creencias muy arraigadas en las sociedades occidentales. Mudde señala:

El hecho de que el cristianismo evangélico juegue en la cultura y en la política de EEUU, un papel más relevante que en Europa puede ser una buena explicación del hecho de que los sentimientos anti establishment en aquel país son más profundos y están más extendidos que en Europa. Mientras que el proceso de democratización y de formación del estado fue dirigido en gran parte de Europa por las élites, basado en una fuerte autoridad central y en una desconfianza de lo popular, en Estados Unidos, el mismo proceso fue implementado por "We the people of the United States", y por una desconfianza en el gobierno central, desconfianza compartida por las masas y por las élites, incluidos los padres fundadores (Mudde, 2010: 4).

Este autor defiende que el creciente éxito electoral de la derecha radical populista se debe a un cambio importante en la forma de hacer política: se dejan de lado los aspectos económicos para insistir en temas y preocupaciones culturales y post-materialistas: corrupción, inmigración y seguridad: 
Los partidos de la derecha radical populista son purificadores que se refieren a una ideología que ha sido traicionada o dilvida por los partidos en el poder, y no tanto profetas que articulan una nueva ideología. No quieren ganar a los votantes para una nueva ideología, sino orientarles hacia un nuevo tema: dejar de lado las cuestiones socioeconómicas como el desempleo y focalizarse en aspectos socioculturales como la emigración. La lucha clave de la derecha radical populista es incrementar la relevancia social de sus preocupaciones: la corrupción, la inmigración y la seguridad (Mudde, 2010: 6)

\section{España 2000, ¿derecha radical?}

El caso de E2000 es representativo por varias razones, ya que durante años se había considerado a España inmune a los extremismos (Casals, 2011) debido a la hostilidad de la sociedad española hacia cualquier recuerdo del régimen fascista, la relativa juventud de la democracia española, el sistema político prácticamente bipartidista (PP y PSOE) o el bajo índice de población inmigrante en España, al menos, hasta principios del año 2000. A pesar de estos factores que podrían inmunizar al país de la extrema derecha, los expertos consideran que en la mayoría de los países europeos existe un potencial electoral suficiente para la emergencia de este tipo de formaciones (Mudde, 2007). Pero éste dependerá de factores relacionados con el contexto social, económico y político e incluso de especificidades territoriales del estado español.

En el caso de E2000, el partido nace en la Comunidad Valenciana en el año 2003. Se autodefine como "populista, social y democrático" y anti inmigración. Su presidente es José Luis Roberto, un empresario vinculado a empresas de seguridad, gimnasio y prostitución que militó en las juventudes franquistas. No obstante, el partido ha buscado posicionarse como una alternativa "no violenta" y democrática según manifiesta su presidente y su programa político. A nivel electoral, E2000 en 2011 se convierte la principal fuerza del espacio político de extrema derecha en número de votos (12.000) en la Comunitat Valenciana. Estos resultados duplicaron las cifras de los comicios de 2007 y quintuplican los de 2003 y 2004.

Además, la formación ha recibido votos en el $95 \%$ de los municipios valencianos y ha conseguido 5 concejales, uno de ellos fuera de la Comunitat Valenciana, concretamente en Alcalá de Henares, la segunda ciudad más poblada de Madrid. Sin embargo, en 2015 el partido perdió 1.810 votos tanto en las elecciones autonómicas como en las locales, entre otros motivos por la posible irrupción en el sistema político valenciano de tres nuevas formaciones, Ciudadanos, Podemos 
y Compromís, que captaron el "voto-protesta" y anti establishment. En el trabajo "La pujanza de los partidos populistas en la Unión Europea: estrategias y apoyos en las últimas elecciones al Parlamento Europeo" (2015) elaborado por Martín Cubas, J., Pavía, J.M, Bodoque A., Veres, E. explican algunas de las causas del apoyo recibido a este último tipo de partidos que presentan un discurso anti establishment y han captado el "voto- protesta". Estos autores destacan por ejemplo:

En relación a las líneas políticas defendidas, hay dos tipos de políticas donde los votantes populistas se diferencian significativamente de los votantes en general: las políticas de inmigración y las políticas de unificación de ta Unión Europea [...] En relación a la integración y la unificación europea también es significativo un mayor rechazo en los votantes populistas con la excepción en este caso de Podemos, Coalición radical de Izquierdas, Ley y Justicia y la Liga Norte [...] En relación a los planteamientos etnonacionalistas, hemos constatado que esta caracterización se corresponde con el rechazo a la inmigración y, por tanto, a favor de políticas que la limiten que es predominante en todos los casos excepto entre los votantes de Podemos y la Coalición Radical de Izquierdas (Martín Cubas et al, 2015: 31).

Su electorado se caracteriza por una diversidad generacional distribuido en dos grandes grupos de edad: jóvenes y jubilados. El primer grupo contiene tanto hombres como mujeres. Como señala Antón Mellón y Hernández Carr en referencia al electorado de las partidos neopopulistas europeos, podríamos considerar que es un electorado con escasa lealtad electoral a partidos tradicionales, poco politizados e ideologizados (salvo los recalcitrantes y creyentes votantes/militantes de derecha radical), individuos que se motivan políticamente por actitudes mucho más primarias: "excesivo" número de inmigrantes y consiguientes cambios en el paisaje urbano, degradación del nivel de vida por la crisis, corrupción, aumento de las desigualdades, inseguridad, protesta anti-establishment" (Antón Mellón y Hernández Carr, 2016: 19)

El segundo grupo, los jubilados, coinciden con un electorado compuesto por individuos de estratos sociales bajos y bajos niveles de educación, ex trabajadores manuales de baja calificación que en el pasado han mantenido algún tipo de relación con el régimen anterior.

Como se ha analizado en el apartado anterior, existe cierta dificultad para catalogar taxativamente a un partido político en la familia de la extrema derecha y especialmente en una de las dos categorías "extrema derecha" o "derecha radical populista" (Mudde). Así, Jens Rydgren (2007: 260) y Cas Mudde (2007: 23) concluyen que ante esta falta de unanimidad, las definiciones de los distintos partidos políticos considerados de "extrema derecha" ("vieja o nueva" segúnlgnazi) o derecha radical populista" (Mudde), tienden a ser arbitrarias y conducir a la confusión, en parte por la influencia de los medios de 
comunicación que relacionan los conceptos de fascistas, neonazis, ultras o radicales.

No obstante, como el tema no es sencillo, creemos que la caracterización más relevante para los propósitos de este estudio, sobre la ideología de extrema derecha del partido político E2000, es la de Cas Mudde. Para examinar a esta formación en este trabajo se extraen e identifican los elementos fundamentales de su discurso la través del documento programático de su campaña electoral en la Comunidad Valenciana en 2015) según los principios que identificarían a una formación política como derecha radical populista.

En primer lugar, como hemos señalado anteriormente, E2000 es un partido de reciente creación (2003) y ha intentado desvincularse de cualquier posible vínculo con el antiguo régimen fascista, como por ejemplo eliminando los símbolos fascistas en sus manifestaciones públicas o cualquier tipo de exaltación al franquismo para evitar su posible asociación con el partido político. Igualmente el presidente ha reiterado la condición democrática de la formación. El partido se define como un partido nuevo; además la formación se declara demócrata y en ningún caso pretende acabar con el sistema, sino que pretende convertirse en una alternativa real a los partidos políticos tradicionales, a los que acusa de corruptos y de ser la causa del declive social, político y económico que ha surgido con la crisis económica de 2007. Además este mensaje se ha realizado desde dentro del sistema ya que el partido forma parte de él y no pretende acabar con la democracia parlamentaria, a diferencia de la extrema derecha.

\section{Nacionalismo exclusionista, racismo cultural y xenofobia}

El partido reivindica el principio de "preferencia nacional" ("los españoles primero"). Una de las consecuencias directas de esta preferencia nacional es la existencia de un grupo al que hay que excluir: la población no nativa o inmigrada. Este discurso, aparentemente promovido por un espíritu proteccionista hacia la población de mayor arraigo, contribuye en gran medida a introducir el discurso xenófobo en Europa y a sesgar la mirada hacia la inmigración en la agenda política europea.

Entre las medidas que promueven el partido se encuentra el freno a la migración hacia Europa mediante la suspensión de entrada a inmigrantes tanto regulares como irregulares o la reducción del número de personas inmigradas residentes, proponiendo su segregación y expulsión, prioritariamente de aquellos cuyos fenotipos son diferenciables. Al mismo tiempo, promocionan el derecho de los nativos de vivir según sus costumbres y por lo tanto, a establecerlas como única norma para todos. La formación se aferra en la idea de la 
incompatibilidad cultural, y en consecuencia, la no convivencia ni el intercambio.

El partido no niega el derecho de las personas inmigrantes a buscar un futuro mejor en España, pero deberán aceptar una serie de condiciones: deben ser personas cualificadas y no se deben "aprovechar" del sistema de protección social español. El programa electoral del partido destaca la necesidad del control de la inmigración masiva en Europa y en España. Su hipótesis es que esta inmigración es una amenaza para la identidad y nacionales. Por ello, cuantos más inmigrantes reciba Europa, más se verán amenazados sus valores, cultura y tradiciones ya que más culturas paralelas se establecerán, sin integrarse, dentro del país.

Al mismo tiempo, establecen una relación directa entre la inmigración masiva y el declive económico, la delincuencia y la disminución de las prestaciones sociales. La tesis es que "somos demasiados". Por ello, están a favor de establecer un estricto control de la inmigración, del endurecimiento de la Ley de Inmigración y de la imposición del principio del ius sanguinis (derecho de sangre) para la obtención de la nacionalidad española, con el objetivo de limitar la concesión de la ciudadanía a los descendientes de españoles. Además, los ciudadanos españoles deben tener prioridad sobre los extranjeros en el acceso a los servicios públicos, sobre todo sanidad y educación.

Igualmente, gran parte de su discurso se centra en los inmigrantes musulmanes, un discurso explícitamente islamófobo, ya que los inmigrantes que profesan el Islam son percibidos como la mayor amenaza para la cultura europea y para la identidad española. El partido considera que la brecha cultural entre estos inmigrantes y la sociedad española hace prácticamente imposible su integración plena (la integración es entendida por la extrema derecha como asimilación cultural). Su discurso refuerza la incompatibilidad entre las creencias religiosas islámicas y el sistema legal nacional, argumentando que los musulmanes no serán capaces de respetar valores propios de las democracias europeas, como la libertad individual o la igualdad de género, cuando estos entren en contradicción con sus principios culturales o religiosos.

Por otra parte, el rechazo a la inmigración masiva está íntimamente ligado con su rechazo al sistema capitalista ya que produce enormes desigualdades entre países, lo que motiva grandes desplazamientos poblacionales desde los países subdesarrollados o en vías de desarrollo hacia Europa, y debido a esta afluencia de extranjeros nuestra cultura y valores europeos se encuentra bajo amenaza. Así, justifican su oposición a la inmigración como consecuencia del sistema capitalista al que también se oponen y ofrecen soluciones para contribuir al desarrollo de los países del Tercer Mundo como modo de evitar la inmigración masiva hacia Europa. 
En síntesis, el viejo racismo ha sido sustituido por ideas de supervivencia cultural y social, por la idea de la pérdida de identidad nacional (soberanía), pérdida de identidad cultural y por el miedo a repartir poco entre muchos. El concepto de "raza" ha sido sustituido por el concepto de "cultura" donde se clasifican los individuos y se diferencian los grupos. Y esta idea de identidad, sobre todo la "cultural", es identificada con el concepto de "nación".

En la misma línea, su discurso en defensa del medio ambiente se relaciona con el principio de defensa de la identidad nacional. Así, aparecen importantes medidas para la protección medioambiental, que se asocia a la necesidad de preservar los recursos y la herencia histórica y cultural del pueblo español.

\section{Estado fuerte y aversión a procesos democráticos}

El partido critica fuertemente el Estado de Bienestar y aunque promueve el liberalismo económico, demanda medidas proteccionistas para el ámbito nacional. Defiende la intervención del Estado en diversas materias, como el aumento del control policial, la expulsión de inmigrantes como medio para garantizar la seguridad, y la garantía de prestaciones sociales para los nacionales. Es lo que Griffin entiende como "liberalismo etnocrático" (2007: 12). Se trata de una concepción política de Estado fuertemente centralizada: el Estado debe ostentar todas o la gran mayoría de las competencias administrativas; el poder y las competencias políticas de las Comunidades Autónomas debe ser muy restringido. Por ello, apuntan a la total abolición del Estado de las Autonomías. El separatismo y los movimientos a favor del independentismo son considerados serias ofensas contra la unidad nacional.

Al mismo tiempo, el partido reconoce la importancia de los niveles local y regional y defiende el mantenimiento y la preservación de la cultura, identidad y tradición española y valenciana como elementos que aportan riqueza a la nación española. Sobre esta cuestión el partido, en sus primeros años, explotó el discurso anti catalanista ${ }^{3}$. Al respecto, Casals Messeguer profundiza en los posibles antecedentes históricos de la "extrema derecha valenciana" y las principales líneas discursivas:

desde finales del franquismo ta ciudad (Valencia) y su hinterland han conformado una placa tectónica ideológica al establecer una frontera física y lingüística con el catalanismo, a menudo asociado a un afán de dominio "imperialista» y marxista. Esta situación ha generado una

\footnotetext{
3 El partido boicoteó la presentación del libro de Vicent Flor, Noves Glories a Espanya sobre anticatalanismo en la Comunidad Valenciana. Como recoge Flor en su libro, E2000 ha lanzado mensajes como "catalanistas terroristas". Más información en http://www.elmundo.es/elmundo/201 1/07/05/valencia/1309891348.html
} 
Ultraderecha española de limitada ideologización y marcado carácter combativo, muy visible en la calle y con fluidos vínculos con los círculos del mundo ublaven y el populismo anti catalanista que éste ha conformado (Casals Messeguer, 2013: 8)

La formación busca conectar con amplios sectores de la sociedad, tanto en la izquierda como en la derecha, interpelando a la clase obrera y a la clase media.

Sobre las cuestiones económicas, no sólo condenan el gran capitalismo, sino también los efectos de la mundialización y globalización por lo que implica para la economía española: la huida de empresas nacionales a países menos desarrollados y la afluencia masiva de mano de obra barata. Exigen la devolución de la soberanía a los estados nacionales y revertir el proceso de integración europeo, pero no proponen la salida directa de España, sino la construcción de una "Confederación de Estados" Europeos con un mayor grado de soberanía para los países miembros. Se unen al denominado movimiento anti europeísta.

Como culpables de esta situación identifican a la derecha liberal y àlos partidos socialdemócratas. Con un mensaje directo y constante, apelan al desencanto de la sociedad frente a los políticos tradicionales y el vacío que estos han ido dejando en importantes capas de la sociedad. Se presentan como "salvadores" del declive social, político y económico que ha surgido con la crisis económica de 2007:

Son los demiurgos de los déficits democráticos, los problemas sociales y las angustias y rencores de sectores de la población huérfanas de identidad política y que escuchan las sirenas ninistas (ni de derechas ni de izquierdas) (Antón Mellón, 2011: 75)

Como se comentaba anteriormente, el partido no se declara contrario a la democracia pero sí muestra cierta hostilidad hacia el sistema de elección de candidatos y por ello, apuesta por un poder ejecutivo dotado de amplios poderes, a menudo por regímenes presidencialistas, y por el recurso a la democracia directa en forma de consultas a los ciudadanos sobre cuestiones que estos partidos consideran relevantes. Estas similitudes argumentales-ideológicas del partido con las propuestas por Cas Mudde sobre la derecha radical populista permiten entender el tipo de formación que es España 2000. No obstante, existen otras dimensiones de los partidos que no son objeto de este estudio, vinculados al estilo y la organización pero que, al menos deben ser destacados, ya que permiten entender mejor la naturaleza política del partido.

Se trata de la figura del presidente, José Luis Roberto, que se asemeja a la figura de un líder carismático e incluso autoritario. Roberto ejerce un fuerte control de la formación, con una presencia indiscutible en cada acción política, mediática y económica. Además Roberto no sólo es el líder del partido, sino que es el dueño de empresas de seguridad, 
academias y gimnasios en la que sus trabajadores o clientes son también sus militantes. De esta manera, el control es todavía mayor sobre la organización. Además, como otros líderes de derecha radical populista, Roberto recurre a un lenguaje soez y comportamiento estridente (Erra y Serra, 2008) como hicieron también Josep Anglada. Jean Marine Le Pen, Umberto Bossi, o el griego Nikolaos Mijaloliakos que obligó a los periodistas a recibirle en pie (Diario Vasco, 2012). Se trataría de la figura de un "líder carismático de camarilla", según el análisis sobre líderes de Eatwell (2007), y cuya figura tiene efectos en el interior del partido, con un núcleo duro de seguidores, con altos grados de lealtad y voluntad por realizar esfuerzos para su causa.

En cuanto a la estructura del partido, tiene una organización jerárquica y piramidál. En la cúspide se encuentra su Presidente y sus 26 vocales. En el segundo escalón están las "Juntas" que se distribuyen de acuerdo a un criterio territorial y son de dos tipos: juntas provinciales (Valencia, Alicante y Castellón) y locales ( 29 en la ciudad de Valencia, 7 en Alicante y 5 en Castellón). En la parte inferior de la pirámide y desde el ámbito local, el partido se organiza en las milicias, escuadras y centurias que se constituyen por dos motivos: el número de personas y las afinidades lúdico- deportivas y religiosas.

El partido tiene democracia interna para la elección de su presidente y su junta directiva y se compone de tres tipos integrantes: los militantes que pagan una cuota mensual de 10 euros, los militantes-simpatizantes que están exentos de estas cuotas por su peso político o histórico y los simpatizantes, que no pagan pero que tienen un carnet y participan en actividades.

Además, algunos de los militantes e incluso un concejal ha sido imputado en una operación de propaganda y difusión de material neonazi y armas, la operación Panzer (El Pais, 2014). José Luis Roberto ha sido denunciado por diversas organizaciones, como SOS Racismo, por incitar al odio racial, la violencia y la discriminación, permitiendo que en sus manifestaciones sean coreados lemas xenófobos y racistas, así como por la exhibición de emblemas nazis y fascistas (Levante, 2013). Por la tanto, los principales argumentos del programa de E2000 coinciden con los parámetros atribuibles a la Derecha Radical Populista, pero, $\dot{e}$ es posible homologar totalmente su discurso al de los partidos neo populistas europeos?

\section{Similitudes entre el programa político de E2000 y los partidos neopopulistas europeos}

La anterior descripción del discurso de E2000 revelaba un partido con planteamientos ideológicos similares a lo que Cas Mudde calificó de "derecha radical populista" en cuestiones como la concepción de un Estado fuerte o un nacionalismo que destaca el derecho a la diferencia 
hacia los inmigrantes. A partir de esta aproximación a la ideología del partido y su categorización como derecha radical populista, nos planteamos si el discurso de la formación valenciana tiene otros elementos en común con los partidos neopopulistas europeos.

Para intentar cuantificar el grado de similitud con estas formaciones, se aplica el modelo elaborado por Antón Mellón (2011), que construye un árbol conceptual de los discursos xenofóbicos de los partidos neopopulistas de la "derecha radical" europea estructurado en tres apartados: liberalismo efnocrático, catastrofismo y populismo.

El trabajo considera que estos tres factores son homogeneizadores culturales del discurso neopopulista sobre la inmigración: un nuevo racismo diferencialista con una base cultural y no ideológica; los enfoques políticos se apoyan en un ultranacionalismo palingenésico (renacimiento de la patria en peligro) y, por último. la propuesta política fundamental reside en los criterios de exclusión ultraidentitaria (un ultranacionalismo excluyente) y la preferencia nacional xenofóbica [xenófoba] para todos los individuos legalmente considerados miembros de la comunidad nacional (Mellón, 2011: 72)

Para realizar esta comparación, se ha aplicado la metodología cuantitativa y se ha codificado la documentación considerando los términos - y sus sinónimos- del modelo de árbol conceptual de Antón Mellón como variables cuantificables $y$, así se han identificado y evaluado los términos comunes que utiliza el discurso de E2000.

El análisis de los resultados permite afirmar que España 2000 asume un discurso que se identifica mayoritariamente con los parámetros de la derecha radical neopopulista europea. En la Tabla 1 del anexo se comprueba que casi de la mitad de los términos utilizados por E2000 en el programa electoral se corresponden con el término catastrofismo, el $40 \%$ con el denominado liberalismo-etnocrático, y en menor medida el populismo (el 14\%). El cómputo general nuestra que el $75 \%$ de los conceptos del programa se relacionan en mayor o en menor grado con el de los partidos neopopulistas europeos.

Sobre el factor homogenizador "liberalismo-etnocrático", se ha comprobado que existe una sobre utilización del rechazo al multiculturalismo, manifestando la imposibilidad de la convivencia de culturas en un mismo territorio y destacando la animadversión hacia el colectivo de inmigrantes musulmanes. Además, califican esta religión de atrasada culturalmente y machista.

Asimismo, vinculan la inmigración con el terrorismo, el aumento del paro, la delincuencia y la pérdida de ayudas sociales de los nacionales en favor de los inmigrantes. Algunos ejemplos del uso de estos conceptos en el discurso de E2000 son: 
Consideramos que la llegada masiva de inmigrantes es una de las causas de elevación del precio de la vivienda que ha repercutido negativamente en nuestra población (España 2000, 2015).

Igualmente reivindica la repatriación de los inmigrantes en paro de larga duración y pretenden cancelar la posibilidad de regularización por arraigo y por reagrupación familiar. El partido presume de fomentar el uso de las señas de identidad como pueblo y propone una sociedad occidental con profundas raíces cristianas a la que defiende ante "un falso progresismo destructor para con lo tradicional" (España 2000, 2015).

Sobre el multiculturalismo, el partido denuncia "el relativismo cultural instalado en nuestra sociedad como una de las peores muestras de la ideología globalizadora" (España 2000, 2015).

En el punto 12 del programa electoral de 2015, titulado "Por una defensa y promoción de las raíces culturales e identitarias de Europa", el partido manifiesta:

Es necesario abordar una amplia tarea educativa: resaltar la naturaleza de las raíces de Europa así como asumir que existen unos sustratos étnicos originarios comunes y unos principios filosóficos fundamentales (el mundo clásico greco-latino y el cristianismo medieval) que configuran una identidad perfectamente definida y delimitada" (España 2000, 2015).

En relación al "catastrofismo", el partido denuncia las consecuencias negativas de la mundialización (paro, deslocalización de empresas) y la combaten mediante el refuerzo de la "identidad nacional", frente al poder hegemónico de la Unión Europea y los Estados Unidos. Este nuevo orden mundial es el origen de problemas como el aumento del paro, de la delincuencia e incluso de la caída demográfica. Sobre esta última cuestión el partido manifiesta:

Luchar contra la doctrina del 'mundialismo a través de la defensa de la soberanía nacional tanto en política, como en economía" (España 2000, 2015).

Manifestamos la necesidad para la sociedad española de defenderse ante la posibilidad de arraigo de un terrorismo islámico llegado con los contingentes migratorios (España 2000, 2015).

Para reforzar la identidad nacional, el partido culpabiliza a los inmigrantes de la posible alteración del supuesto estado natural de la UE.

Finalmente, la UE es responsable de la presencia de 40 millones de inmigrantes en su territorio y de las alteraciones sociales, culturales y laborales que esa presencia masiva ha acarreado [...] en toda Europa se han generado guetos, ha aumentado la delincuencia, los conflictos 
étnico-sociales se suceden en cadena y millones de inmigrantes sin perspectivas laborales recorren el continente subsidiados por los poderes públicos (España 2000, 2015).

En cuanto al "populismo", la formación reitera en su discurso la crítica a los partidos políticos tradicionales por su falta de capacidad para resolver problemas, su alejamiento respecto a los ciudadanos y por la corrupción de las formaciones tradicionales. Señalan que existe una crisis de este sistema y una falta de descrédito de las instituciones. Por este motivo, reivindica el voto al partido como solución para arreglar el sistema, apelando a un cierto sentido común. Con estos argumentos buscan ese electorado que no se identifica ni con los votantes de izquierda ni de derecha (ninistas).Además reclaman la ayuda a organizaciones hermanas para actuar en sinergia y aprovechar estos años decisivos.

Sobre esta cuestión, la formación manifiesta:

Punto 6. La UE es responsable de la pérdida de identidad de Europa [...] Punto 8. La UE es la única responsable de su propio estancamiento" (España 2000, 2015)

La irresponsabilidad de los partidos políticos, su falta de capacidad para el gobierno.... literalmente, han destruido este país. De ahí que haga falta un proyecto de Reconstrucción Nacional [...] (España 2000, 2015).

[...] hace falta que nuestra organización y las organizaciones hermanas estén en condiciones de redoblar sus esfuerzos y actuar en sinergia para aprovechar estos años decisivos (España 2000, 2015)

España 2000 invita a todos los ciudadanos a depositar un voto nulo, un voto en blanco o simplemente no acudiendo a las urnas en señal de protesta por la incapacidad de la clase política española para resolver sus problemas y por la ineficacia de la Unión Europea. Lo que intentamos con este llamamiento es que la sociedad española demuestre su desafección a la actual clase política y deslegitimar los resultados de las urnas" (España 2000, 2015).

\section{Conclusiones}

El ascenso de la derecha populista en Europa occidental es innegable. Sus partidos políticos recogen el descontento de las masas en relación a las élites globalizadoras, la frustración con el establishment político y la estigmatización de los inmigrantes, a los que acusa de ser destructores de la cohesión social y cultural; esta nueva derecha aparece así como baluarte de la recuperación de los valores tradicionales, reproduciendo la estructura social y cultural del estadonación. 
En España, desde el comienzo del siglo XXI, la llegada y residencia de inmigrantes en España y Comunidad Valenciana ha configurado una sociedad diversa cultural y religiosamente. Las formaciones de extrema derecha, tanto tradicionales como las consideradas de derecha radical populista, no han alcanzado un éxito electoral como ha sucedido en otros países europeos.

Sin embargo, la alianza política del partido político E2000 con el Partido por la Libertad y PxC en abril de 2016, así como el acercamiento con otras formaciones radicales europeas $y$ el debilitamiento de las principales fuerzas políticas en la Comunidad Valenciana, han propiciado la reflexión sobre este partido y sus consecuencias e implicaciones políticas en la Comunidad Valenciana.

En relación con este nuevo contexto, se ha articulado la investigación acerca de qué tipo de extrema derecha es la formación política España 2000 y si ésta asume un discurso similar al de los neopopulistas europeos.

El análisis de contenido de su programa político en 2015 según los parámetros de Cas Mudde para identificar a las formaciones de derecha radical, confirma la hipótesis de que el partido asume temáticas y argumentaciones propias de esta corriente política. En este sentido, en su campaña electoral está presente el discurso de reivindicación del principio de preferencia nacional, la exclusión de los inmigrantes o una concepción de estado fuerte y centralizado. Además, E2000 no se cuestiona la democracia parlamentaria, que se asume como tal y participa en ella (o de ella). El partido ataca a los representantes de este sistema, tanto a los políticos como a los partidos políticos tradicionales.

De esta manera, se ha corroborado positivamente la hipótesis inicial: E2000 es un partido político con elementos ideológicos propios de la familia de la "extrema derecha", en concreto se asemeja a la propuesta de "derecha radical populista" de Cas Mudde. Este planteamiento le ha permitido "entrar en el sistema" electoral valenciano de manera muy discreta. Sus 12.000 votos en 2007, 3.300 militantes y 28 sedes en 2016 en la Comunidad Valenciana confirman que no se trata de un fenómeno pasajero ni totalmente marginal.

En segundo término, el análisis empírico ha permitido cuantificar el grado de semejanza del discurso de E2000 con el de las otras formaciones neopoulistas. La conclusión es que la formación adopta elementos propios de los europeos y así se refleja con la sobreutilización en su programa electoral de los conceptos terrorismo, ilegal, seguridad, recursos y su vinculación negativa con inmigración o el excesivo uso de los términos nación, pueblo, recursos y derechos.

Después de este análisis, surgen dos cuestiones: primera, ¿̇estas similitudes ideológicas posibilitarían el éxito de la formación en la 
Comunidad Valenciana o en España?; y segunda, ila sociedad valenciana está totalmente "vacunada" contra los radicalismos?

Las causas de la no emergencia de la extrema derecha en el entorno político valenciano, al menos hasta ahora, tiene componentes tanto endógenos como exógenos y no dependen exclusivamente de su corpus ideológico, que parece tener semejanzas con los neo populistas europeos. Entre los primeros se pueden encontrar aquellos atribuibles al partido y a su incapacidad de encontrar una "fórmula ganadora" capaz de atraer a un número significativo de electores. Las centralidad del discurso en la inmigración tiende a generar una imagen negativa en el público ya que la oferta de los partidos no puede ser monotemática, sino que debe de incluir diversos aspectos de la vida del individuo, incluyendo soluciones concretas y que abarquen las necesidades de la mayor parte de los ciudadanos.

Como veíamos anteriormente, la imagen de E2000 y la de su presidente, así como su presunta relación con grupos racistas y violentos es uno de los elementos que juegan más en su contra. Igualmente, los medios han jugado un papel importante, difundiendo una imagen negativa del partido, con reportajes televisivos, noticias en los periódicos o periodistas infiltrados. Sin embargo, la renovación total de sus líderes para evitar cualquier recuerdo con el pasado fascista e incluso violento será necesaria si pretenden crecer electoralmente, ya que la ciudadanía española tiene una posición moderada e incluso de aversión hacia aquellos gestos que recuerdan al fascismo. Además, dado el sistema político y las características de la sociedad, es muy difícil pronosticar que un líder de E2000 alcance la popularidad de Le Pen, Hofer o Soini.

E2000 ha buscado distanciarse del fascismo y de movimientos como la Falange o el Frente Nacional y aproximarse más al modelo francés, al que considera como una referencia. Así, Jean Marie Le Pen estuvo presente en el acto de creación del partido en 2003 y ha sido considerado una referencia para su presidente.

En este sentido, E2000 se ha dado cuenta de que la única forma de ganar legitimidad hacia el electorado es deshacerse de la etiqueta de fascista y buscar un nicho político en los votantes llamados "ninistas" (ni de izquierda ni de derechas"), el voto anti establishment y, en menor medida, los conservadores, aunque estos constituyen su principal fuente de electores. Por otro lado, la ausencia de un nacionalismo de Estado es uno de los posibles factores que impiden el éxito de la formación.

El componente exógeno se puede dividir en tres aspectos principales: el relativo a la sociedad, el que tiene en cuenta a los partidos mayoritarios y el sistema político que limita $u$ otorga posibilidades para el desarrollo de alternativas. En primer lugar, como hemos comentado, la sociedad española todavía tiene presente el recuerdo de la dictadura y provoca 
una aversión hacia símbolos e imágenes del régimen franquista, un sentimiento que los ciudadanos relacionan con la explotación excesiva del sentimiento de identidad nacional.

Por otro lado, el sistema electoral español tanto los de Cortes Generales y locales, no facilita la entrada de nuevos partidos en el arco parlamentario y, en concreto, la Ley Electoral Valenciana dificulta la representación de los partidos políticos minoritarios ya que fija una barrera electoral considerada alta (el 5\%).

En cuanto al espectro político, sobre todo del lado de la derecha, no está tan fragmentado como en otros países (Finlandia, Italia, Francia, Holanda, Austria). Incluso hasta las elecciones de mayo de 2015, el Parlamento Valenciano se ha caracterizado por el bipartidismo entre PP y PSPV, y además con una mayoría absoluta del partido conservador, el PP, única formación hegemónica del espacio electoral de la derecha. Este hecho conecta directamente con uno de los motivos que apunta Casals Messeguer sobre el fracaso de la extrema derecha española y que explicaría, según Casals, por qué E2000 no alcanzaría tan buenos resultados como otros partidos radicales europeos: el aglutinamiento de los votos radicales en el principal partido conservador, el PP, de parte de la extrema derecha tradicional. Es decir, el cleavage izquierdaderecha se encuentra muy activo en España ya que el PP ocupa todo el espectro derecho en el eje sociocultural, movilizando desde el voto más centrista hasta el de los reducidos nostálgicos falangistas.

¿Puede salir de la marginalidad política y electoral E2000? Aunque todavía es pronto para saber si logrará consolidarse como una fuerza política, como señala Eatwell la demanda del extremismo político está presente en toda Europa y los factores propuestos por los diversos expertos (Eatwell, 2003; Godwin, 2007; Bonet et al., 2006; Cea y Valles, 2009) que pueden propiciar la posibilidad de crecimiento de la demanda electoral de la derecha radical populista se han convertido en una realidad en España y en la Comunidad Valenciana: el debilitamiento de la afección de los ciudadanos hacia los partidos políticos tradicionales, la incertidumbre generalizada en las instituciones europeas, la gestión de la crisis de los refugiados de siria y de los inmigrantes, el temor ante el terrorismo yihadista y la inseguridad ciudadana o las nuevas estrategias discursivas de los partidos de radicales. Elementos que pueden conducir al éxito, enviar a la marginalidad o mantener a E2000 como una opción potencial en el sistema político.

Igualmente, la nueva alianza política del partido con PxC puede contribuir a construir una "mezcla ganadora" en España, aunque la irrupción de nuevos partidos tanto en la izquierda como en la derecha, con discursos anti establisment (Podemos, Compromis y Ciudadanos) y la hegemonía del partido conservador mayoritario en la Comunidad 
Valenciana y España (PP) pueden socavar las posibilidades de éxito de esta coalición o del partido en solitario.

No obstante, aún es pronto para ignorar la presencia de la formación y considerarla como perdedora política. Como se ha señalado, la sociedad europea se encuentra inmersa en un momento de incertidumbre e inseguridad política, incapaz de resolver los problemas derivados de la crisis de los refugiados surgida a raíz de la Guerra de Siria de 2015, como su integración en las sociedades europeas y los posibles brotes de xenofobia y racismo que se han evidenciado en Francia o Alemania (2016), donde se han instalado en campamentos o zonas de acogida. Además de la permanente amenaza del terrorismo yihadista que fomenta actitudes antiinmigración y xenófobas. En este contexto, las opciones neopopulistas han sido capaces de articular discursos que satisfacen las demandas de los ciudadanos frente a los partidos políticos tradicionales.

De cualquier forma, los próximos años indicarán la prevalencia de la derecha radical populista en la Comunidad Valenciana y si ésta ha canalizado las demandas de la sociedad, ya que el partido se encuentran en un momento de "metamorfosis" que persigue legitimarse ante la sociedad valenciana, desvinculándose del fascismo y ofreciéndose como única solución viable para el pueblo. Aunque es casi una condición indispensable para su crecimiento y consolidación, la renovación de sus líderes y la construcción de una oferta más elaborada, más allá del discurso anti inmigración y la identidad nacional y que abarque las necesidades de la mayor parte de los ciudadanos.

\section{Bibliografía}

Antón Mellón, Joan (coord.) (2011). Inmigración y discurso electoral en las elecciones municipales de 2007 en Cataluña. Análisis de la similitud y divergencia en relación al discurso xenófobo de la derecha radical europea neopopulista. Revista Crítica Penal y Poder, 1, 69-87

Antón Mellón, Joan y Hernández Carr, Aitor (2016). El crecimiento electoral de la derecha radical populista en Europa: parámetros ideológicos y motivaciones sociales. Política y Sociedad, 53, 17-28

Betz, Hans George (1994). Radical Right-Wing Populism in Western Europe. Londres: Macmillan.

De Lange, S y Mudde. Cas (2005). Political extremism in Europe. European PoliticalScience, 4, 476-88.

Casals, Xavier (1995). Los neonazis en España. Madrid: Grijalbo 
Casals, Xavier (2003).Ultrapatriotas. Extrema derecha y nacionalismo de la guerra fría a la era de la globalización. Barcelona: Crítica

El Pais (2014). Los jueces absuelven al grupo neonazi que almacenó armas y un lanzagranadas. El Pais Descargado de http://ccaa.elpais.com/ccaa/2014/07/29/valencia/1406644268_563134. html

España 2000 (2015). Programa político. España 2000. Descargado de http://espana2000.org/?page_id=41

España 2000 (2015). Ante las Elecciones Generales del 20 de Diciembre de 2015. España 2000. Descargado de: $\mathrm{http}: / /$ espana2000.org/? $p=3601$

España 2000 (2015). Se preparan las bases para conformar la alternativa patriótica en España. España 2000. Descargado de:http://espana2000.org/?p=3729

España 2000 (2016). La resaca del Breixit. España 2000. Descargado de $\mathrm{http}: / /$ espana2000.org/? $\mathrm{p}=4070 \#$ more -4070

Europa Press (2011). El TS desestima el recurso del Bloc contra la barrera electoral del $5 \%$ de las elecciones de 2007. Europa Press. Descargado de http://www.europapress.es/comunitat-

El Mundo (2011). La ultradreta boicoteja la presentació d'un llibre sobre l'anticatalanisme a València. El Mundo. Descargado de http://www.elmundo.es/elmundo/2011/07/05/valencia/1309891348.html

Gallego, Ferran (2005). Nostalgia y modernización. La extrema derecha española entre la crisis final del franquismo y la consolidación de la democracia (1973-1986). Historia contemporánea, 4, 245-265

Gil, Joaquin (2012). Ultras con piel de ONG. El Pais. Descargado de http://ccaa.elpais.com/ccaa/2012/11/17/valencia/1353176811_374796.

html

Hernández Carr, Aitor (2011). La derecha radical populista en Europa: discurso, electorado y explicaciones. Revista Española de Investigaciones Sociológicas, 136, 141-160.

Hernández Carr, Aitor (2011). La hora del populismo? Elementos para comprender el éxito electoral de Plataforma per Catalunya. Revista de Estudios Políticos, 153, 141-16.

Ignazi, Piero (1993). Extreme Right Parties in Western Europe. Oxford:

Oxford University Press.

Lubbers, M., y P. Scheepers (2002). Extreme right-wing voting in Western Europe. European Journal of Political Research, 41, 345-78.

Martín Cubas, J, Pavía, JM, Bodoque A, Veres, , (2015). La pujanza de los partidos populistas en la Unión Europea: estrategias y apoyos en las 
últimas elecciones al Parlamento Europeo",XII Congreso de la AECPA, 2015.

Movimiento contra la Intolerancia (2014). Informe Especial Raxen. Europa en Crisis: Tolerancia o Barbarie: Racismo, Xenofobia e Intolerancia en Internet. Madrid: Movimiento contra la Intolerancia,

Mudde, Cas (2000). The Ideology of the Extreme Right. Manchester: University Press,

Mudde, Cass (2007). Populist Radical Right Parties in Europe. Manchester: University Press,

Norris, Pippa (2005).Derecha radical populista. Votantes y partidos políticos en el mercado Electoral. Madrid: Akal,

Paniagua Fuentes, J. y Piqueras, J.A (2005). Diccionario Biográfico de Políticos Valencianos, 1810- 200. València: Institut Alfons el Magnànim

Perrineau, Pascal (2005). Does Lepenism exist without Le Pen?. En X. Casals (ed.), Political survival on the extreme right. European movements between the inherited past and the need to adapt to the future (pp. 1231). Barcelona: Institut de Ciències Polítiques i Socials,

Rius, Xavier (2012). Mapa incompleto de la extrema derecha. Blog Xavier Rius. Descargado de http://xavierrius.blogspot.com.es/2012/08/mapa-incompleto-de-la-extremaderecha-2.html

Rydgren, Jens (2007). The sociology of the radical right.: Annual Review of Sociology, 3, 241-262

Rodríguez, José Luis (2006). De la vieja a la nueva extrema derecha (pasando por la fascinación por el fascismo. Historia Actual Online, 9, 21-35.

Sartori, Giovanni (1976). Parties and Party Systems: A Framework for Analysis. Cambridge: Cambridge University Press

Simón, Miguel Ángel (2007). La Extrema Derecha en Europa desde 1945 a nuestros días. Madrid: Tecnos,

Taguieff, Pierre-André (ed) (1993). From Race to Culture: The New Right's View of European Identity. Telos, 98, 99-125.

Wieviorka, Michel (1992). El espacio del racismo. Barcelona: Paidós Ibérica 